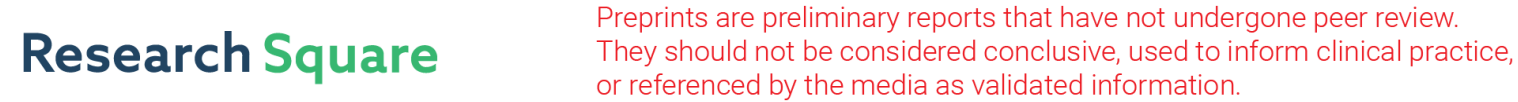 \\ Effect of Workpiece Rotational Speed on Surface Quality in YAG Double-sided Planetary Lapping With the Trajectory Method
}

Yang Lei

Dalian University of Technology

Guo Xiaoguang

Dalian University of Technology

Kang renke

Dalian University of Technology

Zhu Xianglong ( $\square$ zhuxianglong@163.com )

Dalian University of Technology

Jia Yufan

Dalian University of Technology

\section{Research Article}

Keywords: Workpiece rotational speed, Double-side planetary lapping, Trajectory uniformity, Trajectory simulation

Posted Date: January 18th, 2022

DOl: https://doi.org/10.21203/rs.3.rs-1260696/v1

License: (a) (1) This work is licensed under a Creative Commons Attribution 4.0 International License. Read Full License 


\title{
Effect of workpiece rotational speed on surface quality in YAG double-sided planetary lapping with the trajectory method
}

\author{
Lei Yang, Xiaoguang Guo, Renke Kang, Xianglong Zhu*, Yufan Jia
}

Key Laboratory for Precision and Non-traditional Machining Technology of Ministry of Education, Dalian University of Technology, Dalian 116024, PR China

\section{Xianglong Zhu ${ }^{1, *}$, Corresponding author}

Telephone: +86-(0)411-84707430

Fax: +86-(0)411-84707430

E-mail address: zhuxianglong@dlut.edu.cn

\section{Abstract}

The double-sided planetary lapping technology with fixed grit is a good candidate to process thin $\mathrm{Y}_{3} \mathrm{Al}_{5} \mathrm{O}_{12}(\mathrm{YAG})$ crystals wafer. However, the influence of workpiece rotational speed $n_{\mathrm{w}}$ in the planetary wheel on grit trajectory distribution and surface quality has not been revealed, which is of most significance to obtain a better surface workpiece. To solve this problem, the mathematical model for calculating the grit trajectory left on the workpiece in double-side lapping considering the workpiece rotation is established, and a variation coefficient for uniformity of the track point distribution (NUTPD) is introduced to evaluate the trajectory uniformity. Then, the trajectory of multiple grits and the NUTPD value at different workpiece rotational speeds were simulated in MATLAB software. The simulation result showed that the workpiece rotational speed had an extraordinary effect on the uniformity of the motion trajectory leaving on the workpiece surface. The preferred workpiece rotational speed values for lapping are $n_{\mathrm{w}}=1,3,5,7,9$. By conducting a series of tests on the YAG wafer at two typically selected rotational speeds, the surface roughness $\mathrm{Ra}$ and the peak-to-valley (PV) error of flatness were used to characterize the uniformity of surfaces quality. The experimental results demonstrate that there was a more uniform lapping effect, when $n_{\mathrm{w}}=5 \mathrm{rpm}$, and the Ra and PV were $147 \mathrm{~nm}, 2.62 \mu \mathrm{m}$, and $141 \mathrm{~nm}, 2 \mu \mathrm{m}$ at the upper and lower surface after lapped, which was smaller. They were $161 \mathrm{~nm}, 3.05 \mu \mathrm{m}$, and $149 \mathrm{~nm}, 2.74$ $\mu \mathrm{m}$ after lapping when $n_{\mathrm{w}}=6 \mathrm{rpm}$. The experimental result is consistent with the simulation result, and the feasibility and validity of the trajectory method for controlling workpiece surface quality on the planetary double-side lapping were verified.

Keywords: Workpiece rotational speed, Double-side planetary lapping, Trajectory uniformity, Trajectory simulation 


\section{Introduction}

Thin $\mathrm{Y}_{3} \mathrm{Al}_{5} \mathrm{O}_{12}$ (YAG) crystals wafer was a widely used solid-state laser medium in the field of industrial processing, military technology, and scientific research which require high flatness and surface roughness[1]. However, owing to its large diameter-to-thickness ratio, the thin crystal wafer has extremely poor rigidity. Therefore, single side lapping may cause clamping deformation and processing deformation of the workpiece due to the uneven release of the internal stress of the chip, traditionally[2, 3]. In addition, there are still many disadvantages such as low efficiency and uncontrollable uniformity in free grit lapping[4, 5]. Double-sided planetary lapping technology with a fixed abrasive grinding pad that has a high removal rate, controllable uniformity, and that relieves uneven release of internal stress of wafer surface, which can solve many problems as mentioned above[6,7]. Thus, double-sided planetary lapping technology with a fixed abrasive grinding pad can be developed for lapping for the YAG wafer. The surface quality such as flatness and surface roughness is significantly influenced by the uniformity of grit trajectory left on the wafer surface[8].

In recent years, as an effective method, kinematic and trajectory analysis was commonly used to analyze workpiece surface quality after lapping and polishing. Teng et al.[9] attempted to investigate the influence of the mean velocity at the whole workpiece surface on the uniformity of the removal rate and the relationship between the kinematic parameters and the removal rate was given. Furthermore, Kim et al.[10]and Jeong et al.[11] proposed the concept of the sliding distance and kinematic number which is a combination of the basic kinematic parameters, and they found that the sliding distance distribution and the nonuniformity of the workpiece were significantly influenced by the kinematic number. Feng et al.[12] also investigated the sliding distance distribution on the workpiece to evaluate the processing uniformity, and they found the uniformity reaches a maximum when the rotational speed of the workpiece and pad is equal. However, the conclusion mentioned above is commonly derived from free grit lapping and polishing. In fixed grit lapping and polishing, an early study that the workpiece surface quality influenced by grit trajectory distribution is conducted by Uhimann[8]. Further, to obtain a more uniform trajectory distribution on the workpiece surface, many studies[13-22] have been carried out based on trajectory analysis, mainly for designing lapping and polishing tools and optimizing process parameters. In the aspect of processing parameter optimization, it has been demonstrated that the kinematic parameters had a notable influence on the uniformity of the trajectory distribution, and clarified the range of values for better uniformity. However, these studies were focused on the large-size workpiece and one-sided lapping and polishing. Due to the coupling of multiple motions, the trajectory has a higher complexity at double-sided planetary lapping as compared to one-side lapping[23]. Wang et al.[24] analyzed the relative movement between the planetary wheel and lapping pad, and established a trajectory equation of one grit on the planetary wheel to obtain uniform trajectory distribution by the rotation speed optimization. Hashimoto et al.[25] combined kinematic and Preston's law, and estimated the material removal uniformity from the contact pressure distribution and relative velocity distribution in double-side planetary polishing. However, the result above shows the uniformity of the entire planetary gear instead of a single workpiece. King et al.[26] analyzed the movement process of double-sided polishing and established the mathematical model. They demonstrated the existence of workpiece rotation from the theory in double-side planetary polishing. Satake et al.[27] measured the relationship between the upper and lower friction coefficients of the wafer in double-sided polishing and the rotation speed through experiments, and the results 
showed that the friction coefficients of the upper and lower surfaces were inconsistent and mutative. The flatness of the workpiece can be improved by optimizing the rotation speed. However, these research studies did not consider the effect of rotation of the workpiece in the planetary gear on trajectory distribution and uniformity, and the intrinsic relationship between the workpiece rotational speed and the workpiece surface quality after double-side planetary polishing had not been revealed up until now. As, the more uniform trajectory distribution is, the better the surface quality obtained. Therefore, investigating the effect of workpiece rotational speed on uniformity of grit trajectory distribution, which is significant meaning for guiding the improvement of surface quality.

In this study, the mathematical model for calculating the grit trajectory left on the workpiece in double-side lapping considering the workpiece rotation is established. Then, an estimation method for uniformity of the grit trajectory distribution is introduced, and the trajectory of multiple grits on the pad is simulated in MATLAB software. The influence of the workpiece rotation on the uniformity of the grit trajectory distribution is analyzed under a wide range, and the difference in the trajectory distribution on odds and even workpiece speed is revealed. The experiments at two typically selected rotational speeds were carried out to verify the correctness of this model.

\section{Estimation method for grit trajectory distribution}

\subsection{Kinematic model of grit trajectory left on the workpiece surface}

Figure 1 shows that a schematic of the double-side grinding process in a real processing situation, where several workpieces are ground simultaneously. The workpieces have almost the same geometric shape and size, which are in the form of round blocks. They are held by planetary wheel through their round hole, which rotates around its axis under the action of the friction between the upper and lower pad. The planetary wheels rotate around the center of the planetary wheel and revolve around the center of the sun gear driven by the sun gear and the fixed ring gear. The sun gear as well as the upper and lower pad, i.e upper and lower plate, rotates around the center of the respective. However, the rotating direction of the upper and lower pad is opposite, which may achieve better consistency of material removal on upper and lower surfaces.

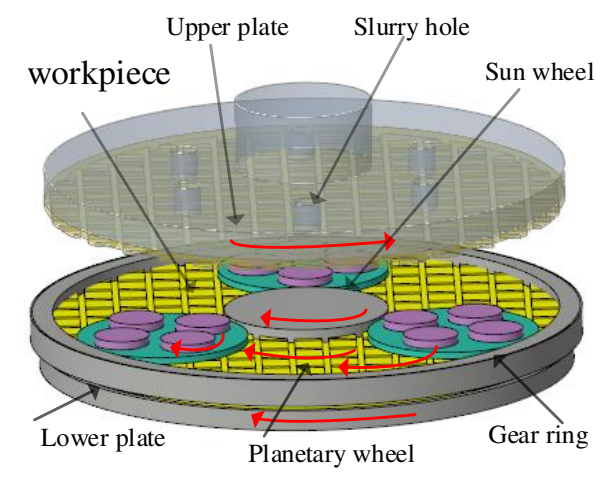

Fig.1 The schematic diagram of a double-side planetary lapping

Figure 2 shows the kinematic diagram. It is assumed that the surface of the upper and lower pad, as well as workpieces, are flat, and the pad is divided into many grid-like blocks which contain abundant grit. The grit on the pad is considered to be individual points of equal height evenly distributed on a layer. moving coordinate systems $\mathrm{x}_{1} \mathrm{O}_{1} \mathrm{y}_{1}, \mathrm{x}_{2} \mathrm{O}_{2} \mathrm{y}_{2}, \mathrm{x}_{3} \mathrm{O}_{3} \mathrm{y}_{3}$ and $\mathrm{x}_{4} \mathrm{O}_{4} \mathrm{y}_{4}$ are located at the 
workpiece center, the planetary wheel center, the pad center, and the $q$ position respectively. Fixed coordinate systems $\mathrm{x}_{0} \mathrm{O}_{0} \mathrm{y}_{0}$ which are called base coordinates are located at the workpiece center. Point $q$ is regarded as a fixed grid on the pad. L is the distance from point $q$ to the center of the pad, and the initial angle between line $\mathrm{o}_{3} q$ and $\mathrm{x}_{2}$ axis for the point $q$ is $\alpha$. The $e$ is the distance from the center of the pad to the center of the planetary wheel. The distance from the center of the planetary wheel to the center of the workpiece is $d$. The sun wheel and the pad rotations are $n_{\mathrm{s}}$ and $n_{\mathrm{p}}$ respectively. The planetary wheel rotational speed around its own center is $n_{\mathrm{ro}}$ with revolving speed are around the center of the sun gear. The workpieces rotate at a speed of $n_{\mathrm{w}}$. After $\mathrm{t}$ seconds, the pad rotates at an angle of $\theta_{3}$, the planetary wheel rotates at an angle of $\theta_{2}$ with revolves at an angle of $\theta_{\mathrm{h}}$, and the workpieces rotate at an angle of $\theta_{1}$. The number of teeth of the sun gear, the planetary wheel, and the fixing ring gear is $z_{\mathrm{s}}$, $\mathrm{Z}_{\mathrm{p}}$, $\mathrm{Z}_{\mathrm{f}}$ respectively, which is determined once the structure of the processing equipment is determined.

To obtain the grit moving trajectory on the workpiece, i.e under the coordinate systems $\mathrm{x}_{3} \mathrm{O}_{3} \mathrm{y}_{3}$, it is necessary to construct kinematic mode of one grit based on mechanism reversal method and D-H coordinate conversion method. The central axis of the planetary wheel is considered stationary. According to the mechanism reversal method for gear system, the relative change of rotation angle of the sun gear and the pad is $\theta_{\mathrm{h}}$ which means the sun gear and the pad rotates slower $\theta_{\mathrm{h}}$, or the pad rotates faster $\theta_{\mathrm{h}}$ when the rotational direction between the revolution of the planetary wheel and the pad is the same. According to the D-H coordinate conversion, the coordinate system $\mathrm{x}_{1} \mathrm{O}_{1} \mathrm{y}_{1}$ has only the rotation angle $\theta_{1}$ relative to the coordinate system $\mathrm{x}_{0} \mathrm{O}_{0} \mathrm{y}_{0}$; the coordinate system $\mathrm{x}_{2} \mathrm{O}_{2} \mathrm{y}_{2}$ has the rotation angle $\theta_{2}$ and the length $d$ relative to the coordinate system $\mathrm{x}_{1} \mathrm{O}_{1} \mathrm{y}_{1}$; the coordinate system $\mathrm{x}_{3} \mathrm{O}_{3} \mathrm{y}_{3}$ has the rotation angle $\theta_{3}$ and the eccentric distance e relative to the coordinate system $\mathrm{x}_{2} \mathrm{O}_{2} \mathrm{y}_{2}$; the coordinate system $\mathrm{x}_{4} \mathrm{O}_{4} \mathrm{y}_{4}$ relative to the coordinate system $\mathrm{x}_{3} \mathrm{O}_{3} \mathrm{y}_{3}$ has a length $\mathrm{L}$ only. Therefore, the coordinate of point $q$ in the coordinate system $\mathrm{x}_{0} \mathrm{O}_{0} \mathrm{y}_{0}$ can be deduced as follows:

$$
\begin{aligned}
{\left[\begin{array}{l}
x \\
y \\
z \\
1
\end{array}\right] } & =\left[\begin{array}{cccc}
\cos \theta_{1} & -\sin \theta_{1} & 0 & 0 \\
\sin \theta_{1} & \cos \theta_{1} & 0 & 0 \\
0 & 0 & 1 & 0 \\
0 & 0 & 0 & 1
\end{array}\right]\left[\begin{array}{cccc}
\cos \theta_{2} & -\sin \theta_{2} & 0 & d \\
\sin \theta_{2} & \cos \theta_{2} & 0 & 0 \\
0 & 0 & 1 & 0 \\
0 & 0 & 0 & 1
\end{array}\right] \\
& \times\left[\begin{array}{cccc}
\cos \left(\theta_{3}-\theta_{h}+\alpha\right) & -\sin \left(\theta_{3}-\theta_{h}+\alpha\right) & 0 & e \\
\sin \left(\theta_{3}-\theta_{h}+\alpha\right) & \cos \left(\theta_{3}-\theta_{h}+\alpha\right) & 0 & 0 \\
0 & 0 & 1 & 0 \\
0 & 0 & 0 & 1
\end{array}\right]\left[\begin{array}{l}
L \\
0 \\
0 \\
1
\end{array}\right]
\end{aligned}
$$

Then, the trajectory equation of the grit can be simplified as:

$$
\left\{\begin{array}{l}
x \\
y
\end{array}=\left\{\begin{array}{l}
\operatorname{ecos}\left(2 \pi t\left(n_{w}+n_{\mathrm{ro}}\right)\right)+\mathrm{d} \cos \left(2 \pi n_{w} \mathrm{t}\right)+\mathrm{L} \cos \left(\left(2 \pi t\left(n_{w}+n_{\mathrm{ro}}+n_{p}-n_{\mathrm{re}}\right)+\alpha\right)\right) \\
\operatorname{esin}\left(2 \pi t\left(n_{w}+n_{\mathrm{ro}}\right)\right)+\mathrm{d} \sin \left(2 \pi n_{w} \mathrm{t}\right)+\mathrm{L} \sin \left(\left(2 \pi t\left(n_{w}+n_{\mathrm{ro}}+n_{p}-n_{\mathrm{re}}\right)+\alpha\right)\right)
\end{array}\right.\right.
$$

The rotations speed in the equation is a vector, and it is can be defined as the positive when the rotation is clockwise direction.

To obtain the rotation speed and revolution speed of the planetary wheel, it is necessary to analyze the rotational relation among motion mechanisms in the processing system. First, according to the mechanism reversal method, the transmission ratio between the sun gear and the planetary wheel can be obtained as follows: 


$$
i_{s p}=\frac{n_{s}-n_{h}}{n_{r o}-n_{h}}=-\frac{z_{p}}{z_{s}}
$$

Next, the transmission ratio between the fixed gear and sun gear can be deduced as follows:

$$
i_{s g}=\frac{n_{s}-n_{h}}{n_{f}-n_{h}}=-\frac{z_{f}}{z_{s}}
$$

Finally, the rotation speed $n_{\mathrm{ro}}$ and revolution speed are of the planetary wheel can be derived as follows:

$$
\left\{\begin{array}{c}
n_{r o}=\frac{z_{s}\left(z_{p}-z_{f}\right)}{z_{p}\left(z_{s}+z_{f}\right)} n_{s}+\frac{z_{f}\left(z_{s}+z_{p}\right)}{z_{p}\left(z_{s}+z_{f}\right)} n_{f} \\
n_{r e}=\frac{z_{s} n_{s}+z_{f} n_{f}}{z_{s}+z_{f}}
\end{array}\right.
$$

When $n_{\mathrm{f}}$ is equal to zero, the (5) can be simplified:

$$
\left\{\begin{array}{c}
n_{r o}=\frac{z_{s}\left(z_{p}-z_{f}\right)}{z_{p}\left(z_{s}+z_{f}\right)} n_{s} \\
n_{r e}=\frac{z_{s}}{z_{s}+z_{f}} n_{s}
\end{array}\right.
$$

Therefore, the grit trajectory left on the workpiece is calculated to use the following trajectory equation,

$$
\left\{\begin{aligned}
x= & \operatorname{ecos}\left(2 \pi t\left(n_{w}+\frac{z_{s}\left(z_{p}-z_{f}\right)}{z_{p}\left(z_{s}+z_{f}\right)} n_{s}\right)\right)+\mathrm{d} \cos \left(2 \pi n_{w} \mathrm{t}\right) \\
& +\mathrm{L} \cos \left(\left(2 \pi t\left(n_{w}+\frac{z_{s}\left(z_{p}-z_{f}\right)}{z_{p}\left(z_{s}+z_{f}\right)} n_{s}+n_{p}-\frac{z_{s}}{z_{s}+z_{f}} n_{s}\right)+\alpha\right)\right) \\
y & =\operatorname{esin}\left(2 \pi t\left(n_{w}+\frac{z_{s}\left(z_{p}-z_{f}\right)}{z_{p}\left(z_{s}+z_{f}\right)} n_{s}\right)\right)+\mathrm{d} \sin \left(2 \pi n_{w} \mathrm{t}\right) \\
& +\operatorname{Lsin}\left(\left(2 \pi t\left(n_{w}+\frac{z_{s}\left(z_{p}-z_{f}\right)}{z_{p}\left(z_{s}+z_{f}\right)} n_{s}+n_{p}-\frac{z_{s}}{z_{s}+z_{f}} n_{s}\right)+\alpha\right)\right)
\end{aligned}\right.
$$

which is controlled by four processing parameters that are the rotational speed of the sun gear $n_{\mathrm{s}}$, the rotational speed of the pad $n_{\mathrm{p}}$, the rotational speed of the workpiece $n_{\mathrm{w}}$, and the processing time $t$. 


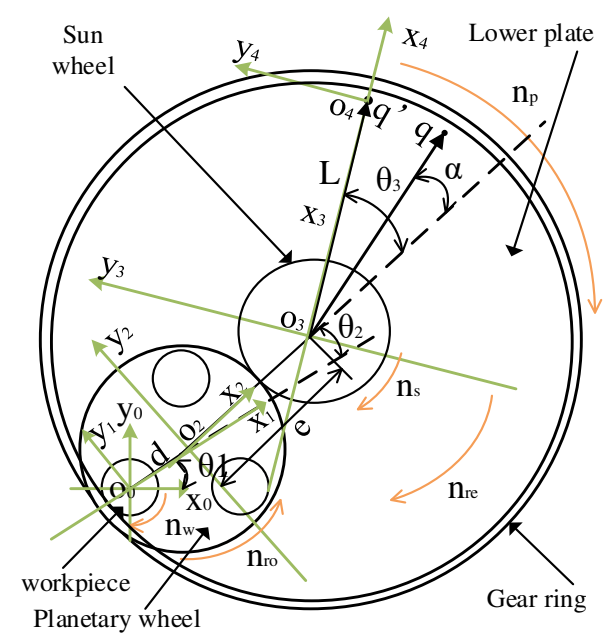

Fig.2 Motion trajectory model of the double-side planetary lapping

\subsection{Evaluation method for grit trajectory distribution uniformity}

As there are numerous grits on the pad, it is difficult to distinguish these complex trajectories on the workpiece. To quantitatively judge whether the grit trajectory on the workpiece is evenly distributed or not, a calculation method of track distribution is applied in this paper.

First, the workpiece surface is divided into $\mathrm{n}$ square regions, as shown in fig.3, and the size of each square region is $1 \mathrm{~mm} \times 1 \mathrm{~mm}$ with recording $\mathrm{s}_{\mathrm{i}}$, and the discretizing time step is set to $0.001 \mathrm{~s}$ to obtain a precise trajectory distribution. Furthermore, the number of track points in the ith square region are record mi, and the density of trajectory point in each square region can be calculated as follows:

$$
\rho_{i}=\frac{m_{i}}{s_{i}}
$$

Then, the average amount of track points in all square regions can be calculated as follows:

$$
u=\frac{1}{n} \sum_{i=1}^{n} \rho_{i}
$$

Finally, NUTPD is applied to represent the uniformity of the track point distribution on the workpiece, which can be expressed as follows:

$$
N U T P D=\sqrt{\frac{1}{n} \sum_{i=1}^{n}\left(\rho_{i}-u\right)^{2}} / u
$$

It is obvious that the smaller value of NUTPD represents the more evenly grit scratches distribution, which can be used to quantitatively predict the surface quality of the workpiece. 


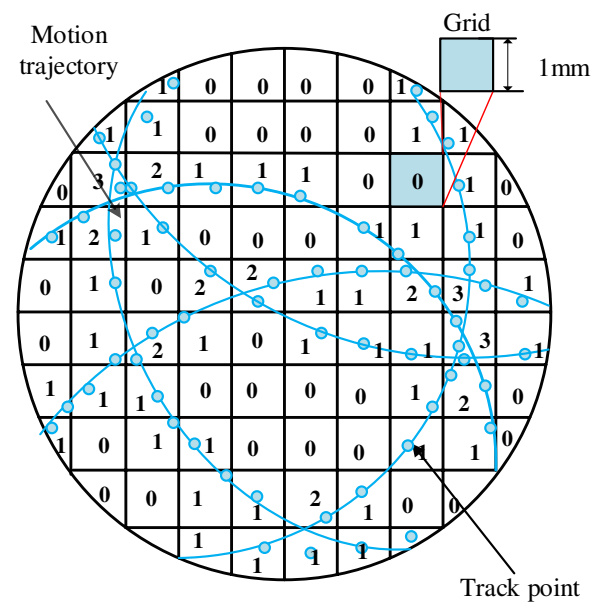

Fig.3 The mesh of the workpiece surface

\section{The simulation of trajectory distribution at different workpiece speeds}

Based on the kinematic model of grit trajectory and the evaluation method for grit trajectory distribution uniformity, the trajectory distribution of the muti-grits left on the workpiece was calculated using MATLAB. To reveal the effect of workpiece rotational speeds on the workpiece surface quality, the grit trajectory distribution of upper and down workpiece surfaces were analyzed under different rotational speeds. In calculation, the workpiece rotational speeds were sited from $1 \mathrm{rpm}$ to $10 \mathrm{rpm}$ with an equal value interval of $1 \mathrm{rpm}$. The rotational speed of the upper and down pad was 20rpm, and their rotational direction was opposite. Taking the workpiece radius $r=7.5 \mathrm{~mm}$, the sun wheel rotational speed $\mathrm{ns}=10 \mathrm{rpm}$, the gear teeth of the sun wheel $\mathrm{z}_{\mathrm{s}}=55$, the gear teeth of the planetary wheel $\mathrm{z}_{\mathrm{p}}=66$, the gear teeth of the fixed ring $\mathrm{Z}_{\mathrm{f}}=190$, and the calculation time 10000 steps. In addition, the inner and outer radius of the pad is 80 and 190 respectively, and the grit block was arranged as a grid in the pad, which the number of the grit was 20736 as shown in Fig.4. The simulation results of the NUTPD for the global trajectory distribution are shown in Fig.5.

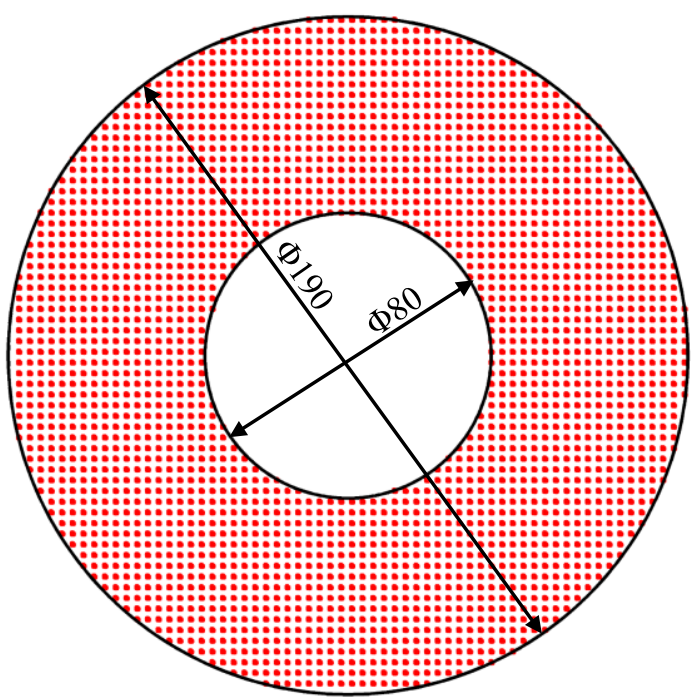

Fig.4 Schematic of distribution of the grit on the pad 


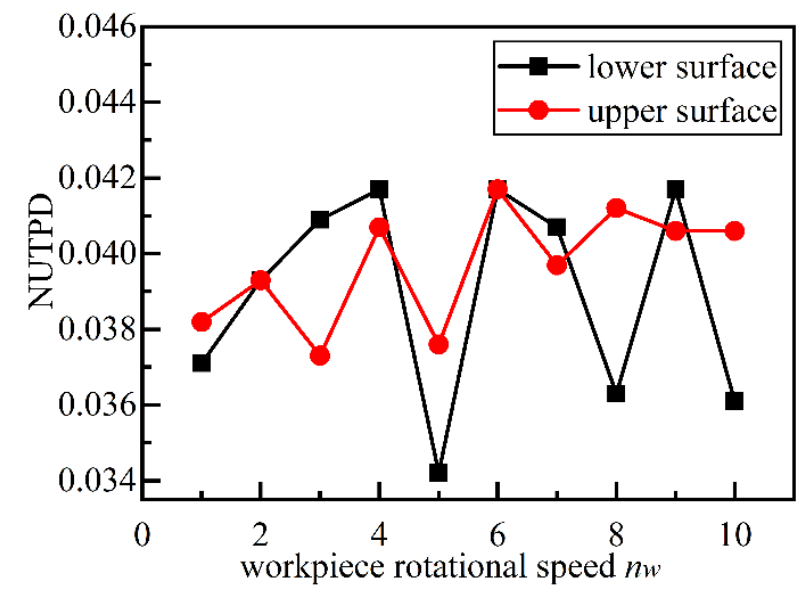

Fig.5 The simulation results at different workpiece rotational speeds

As presented in Fig.5, it can be seen that workpiece rotational speed indeed has a significant influence on the global trajectory distribution of the grit in both upper surface and lower surface. In addition, the effect of the workpiece rotational speed was similar, which the better value of NUTPD can be obtained in the speed selected odds than selected even, except for some special speed because of the opposite rotational direction of the two pads. The curves tend to fluctuate with workpiece rotational speed increases, and the NUTPD ranges from approximately $0.34-0.42$, but the lower surface fluctuates more drastically than the upper surface. The data presented in this figure confirm that the smallest NUTPD value can be obtained when the workpiece rotational speed is $5 \mathrm{rpm}$ on the lower surface, at this time, the upper surface still gets a small value of NUTPD which means better flatness could be obtained.

To further reveal the difference between the effect of odd rotational speed on the trajectory distribution and that of even rotational speed on the trajectory distribution, the profile of the trajectory density left on both surfaces of the workpiece was simulated at two typically selected workpiece rotational speeds: $n_{\mathrm{w}}=5 \mathrm{rpm}, 6 \mathrm{rpm}$.

As Fig. 6 and Fig. 7 show that the workpiece rotational speed $n_{\mathrm{w}}$ has indeed significant influence on the abrasive trajectory distribution, and the trajectory distribution at $n_{\mathrm{w}}=5 \mathrm{rpm}$ is better than the abrasive trajectory distribution at $n_{\mathrm{w}}=6 \mathrm{rpm}$. The average trajectory density ratio of the lower surface, which is the ratio of trajectory density in each squares region to the average amount of trajectory density in all squares region, is about 2250 when $n_{\mathrm{w}}=5 \mathrm{rpm}$, and the variation of trajectory density ratio range from about 2050 to 2450 . In contrast, the average trajectory density ratio of the lower surface is about 2220 when $n_{\mathrm{w}}=6 \mathrm{rpm}$, and the variation of trajectory density ratio range from about 2000 to 2500 . In addition, it can be seen that the area where the abrasive grains deviate from the average number is also less when the rotation speed is 5rpm, compared with the rotation speed of 6rpm. In the same way, the distribution of abrasive grain track points on the upper surface is also more even when the speed is $5 \mathrm{rpm}$. Obviously, the simulation results of trajectory profiles in different rotational speeds are consistent with the NUTPD result in fig5. The track density ratio reflects the number of scratches per unit area of abrasive particles. The uneven number of scratches on the surface of the workpiece will cause inconsistent roughness on the surface, which will affect the flatness of the workpiece. A more uniform scratch distribution helps to achieve better roughness and flatness. Therefore, uniform workpiece surface roughness and flatness can be obtained at an odd rotational speed. Furthermore, lapping 
experiments are carried out on the YJ-6B5LC lapping machine to validate the simulation results.

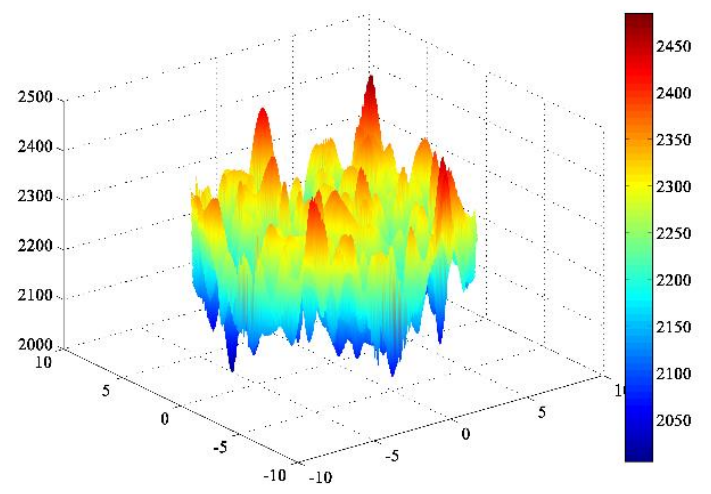

(a)

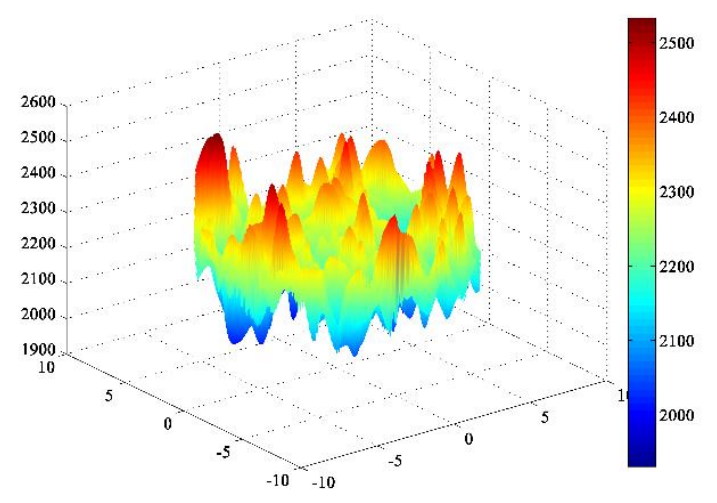

(b)

Fig.6 The grit trajectory distribution cloud map on a lower surface with different rotation speeds. (a) $n_{\mathrm{w}}=5 \mathrm{rpm}$. (b) $n_{\mathrm{w}}=6 \mathrm{rpm}$

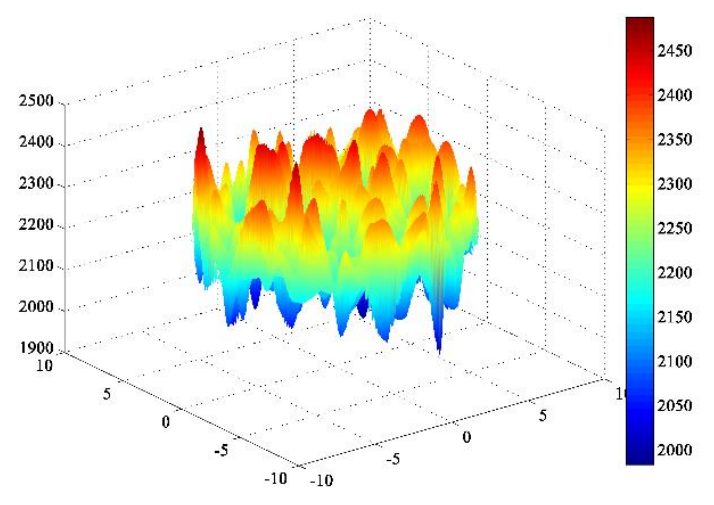

(a) 


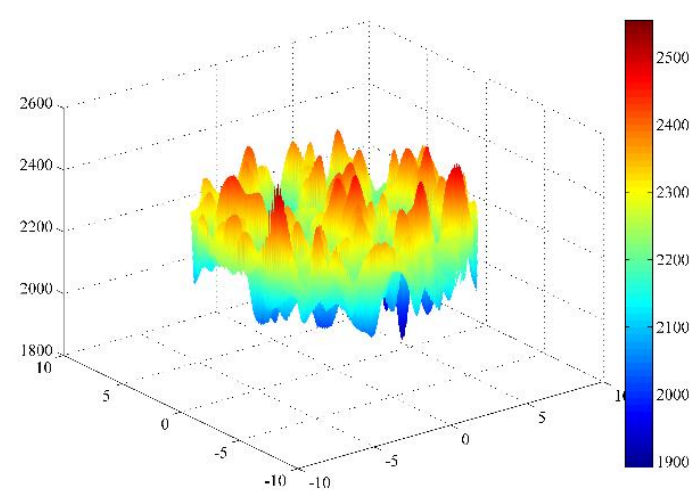

(b)

Fig.7 The grit trajectory distribution cloud map on the upper surface with different rotation speeds. (a) $n_{\mathrm{w}}=5 \mathrm{rpm}$. (b) $n_{\mathrm{w}}=6 \mathrm{rpm}$

\section{Experimental}

The experiments of lapping with the fixed grit diamond pad were conducted on the double-side lapping machine (YJ-6B5LC by Yujing Machinery co., Ltd. Hunan, China) shown in Fig.8. Thin YAG wafer sized $\varphi 15 \mathrm{~mm}$ with an initial thickness of $0.7 \mathrm{~mm}$ and good consistencies of original surface roughness after grinding was chosen as a test sample. Deionized water is chosen as a slurry that can adjust the friction between the workpiece and the polishing pad. The adjustment of the workpiece speed is achieved by adjusting the flow rate of the polishing liquid because it can change the friction between the workpiece and the polishing pad. After measuring the relationship between the coolant flow rate and the rotation angle of the workpiece, it is determined that the coolant flow rate is $100 \mathrm{ml}$ and $115 \mathrm{ml}$ when the rotation speed of the workpiece is 5rpm and 6rpm, respectively, the rotational speed of the pad and the sun gear is consistent with the simulation, the processing time was approximately $45 \mathrm{~min}$.
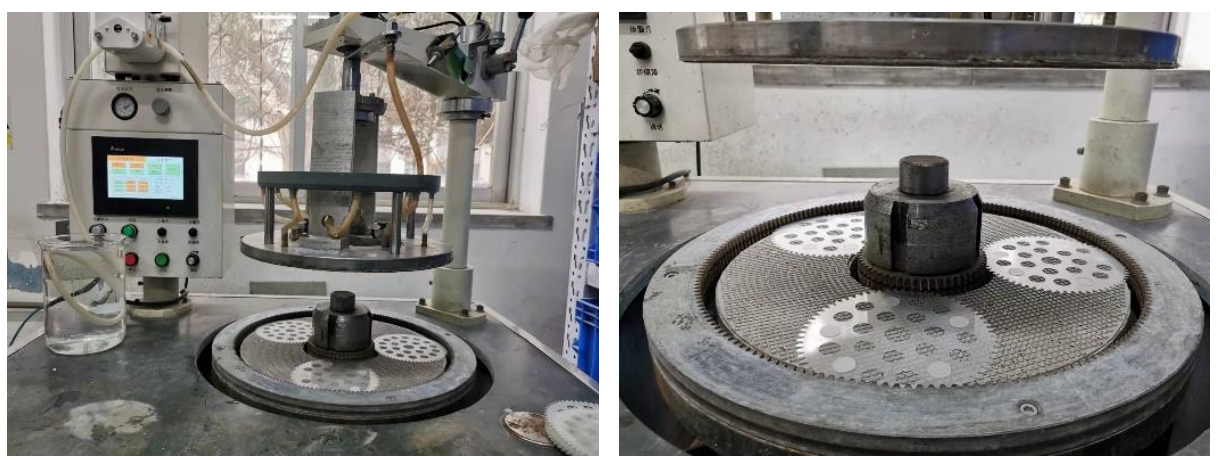

Fig.8 The schematical of experiment with YAG wafer on the double-side planetary lapping

After lapping, Thin YAG wafer flatness (peak-to-valley, PV) was measured by a flatness measuring instrument (Corning FlatMaster 200, USA) and the surface roughness (Sa) was represented by a 3D optical profiler (ZYGO NV5000, USA) at 24 different positions to obtain the profile of roughness distribution. The position of the 24 different testing regions is shown in Fig.9. The variation coefficient of the roughness $(V C R)$ is the ratio of the average value to standard deviation of the roughness, and average values are reported to evaluate surface quality after the process. 


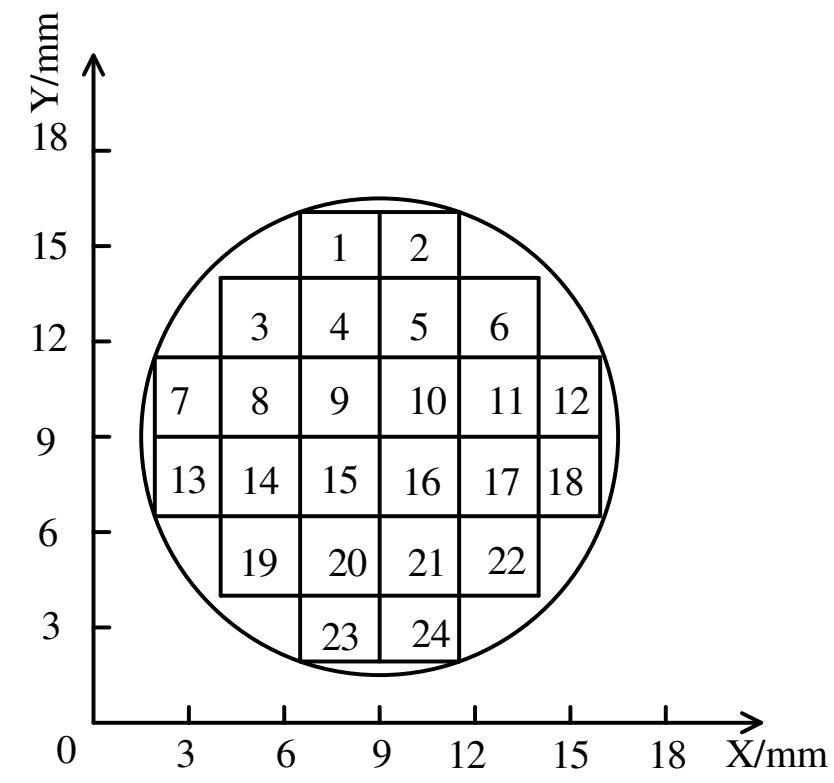

Fig.9 The distribution of the testing location of the roughness on the workpiece surface

\section{Results and discussion}

\subsection{Surface roughness}

The roughness results are shown in Fig.10, Fig.11and Fig.12. Fig.10a and Fig.10b are the roughness distribution of the upper and lower surface at $n_{\mathrm{w}}=5 \mathrm{rpm}$, respectively. Fig.10c and Fig. 10d are the roughness distribution of the upper and lower surface at $n_{\mathrm{w}}=6 \mathrm{rpm}$, respectively. Fig. 11 shows the roughness at selected workpiece rotational speed. The data presented in fig.10 and Fig.11 confirm that the workpiece rotational speed has obviously an extraordinary influence on the roughness. Firstly, the average surface roughness was $147 \mathrm{~nm}$ and $141 \mathrm{~nm}$ in the upper surface and lower surface at $n_{\mathrm{w}}=5 \mathrm{rpm}$, respectively. However, they are $161 \mathrm{~nm}$ and $149 \mathrm{~nm}$ at $n_{\mathrm{w}}=6 \mathrm{rpm}$, respectively. The average surface roughness was small at $n_{\mathrm{w}}=5 \mathrm{rpm}$, which indicated a better lapping effect. The roughness of a center region reached $135 \mathrm{~m}<\mathrm{Ra}<161 \mathrm{~nm}$, while that at the edge was $134<\mathrm{Ra}<158 \mathrm{~nm}$ and the maximum difference value is $30 \mathrm{~nm}$ in the lower surface at $n_{\mathrm{w}}=5 \mathrm{rpm}$. This maximum difference value is $34 \mathrm{~nm}$ on the upper surface at $n_{\mathrm{w}}=5 \mathrm{rpm}$. The maximum difference in surface roughness of the workpiece at $n_{\mathrm{w}}=6$ rpm is greater than that at $n_{\mathrm{w}}=5 \mathrm{rpm}$, and they are $51 \mathrm{~nm}$ and $34 \mathrm{~nm}$ respectively, which also shows the evenness of the workpiece surfaces after lapping was poor at $n_{\mathrm{w}}=6 \mathrm{rpm}$. Combined with the simulation results of the grit trajectory, it is not difficult to find that the experimental results of the surface uniformity of the workpiece are consistent with the simulation results of the uniformity of the abrasive trajectory. A more intuitive comparison result between the experiment and the simulation is shown in Fig.12, and the variation coefficient of the roughness $(V C R)$ and the grit trajectory point density (NUTPD) can reflect the uniformity of the roughness distribution and grit trajectory distribution, respectively. It was found that, when the workpiece rotational speed selected 6rpm, the variation coefficient of the roughness $(V C R=0.069,0.070$ respectively) is rather bigger than that $(V C R=0.063$, 0.051 respectively) of the speed selected 5rpm, which indicates that higher non-uniformity of the surface quality could emerge at $n_{\mathrm{w}}=6 \mathrm{rpm}$. This changed tendency of the variation coefficient of the 
roughness in the experimental result is exactly similar to the tendency of the variation coefficient of the grit trajectory distribution in the simulation, which implied the non-uniformity of the roughness distribution was caused by grit trajectory distribution. Thus, the reliability of the constructed model is verified. Therefore, when the workpiece speed is odd, the surface roughness after machining is smaller than when it is even.
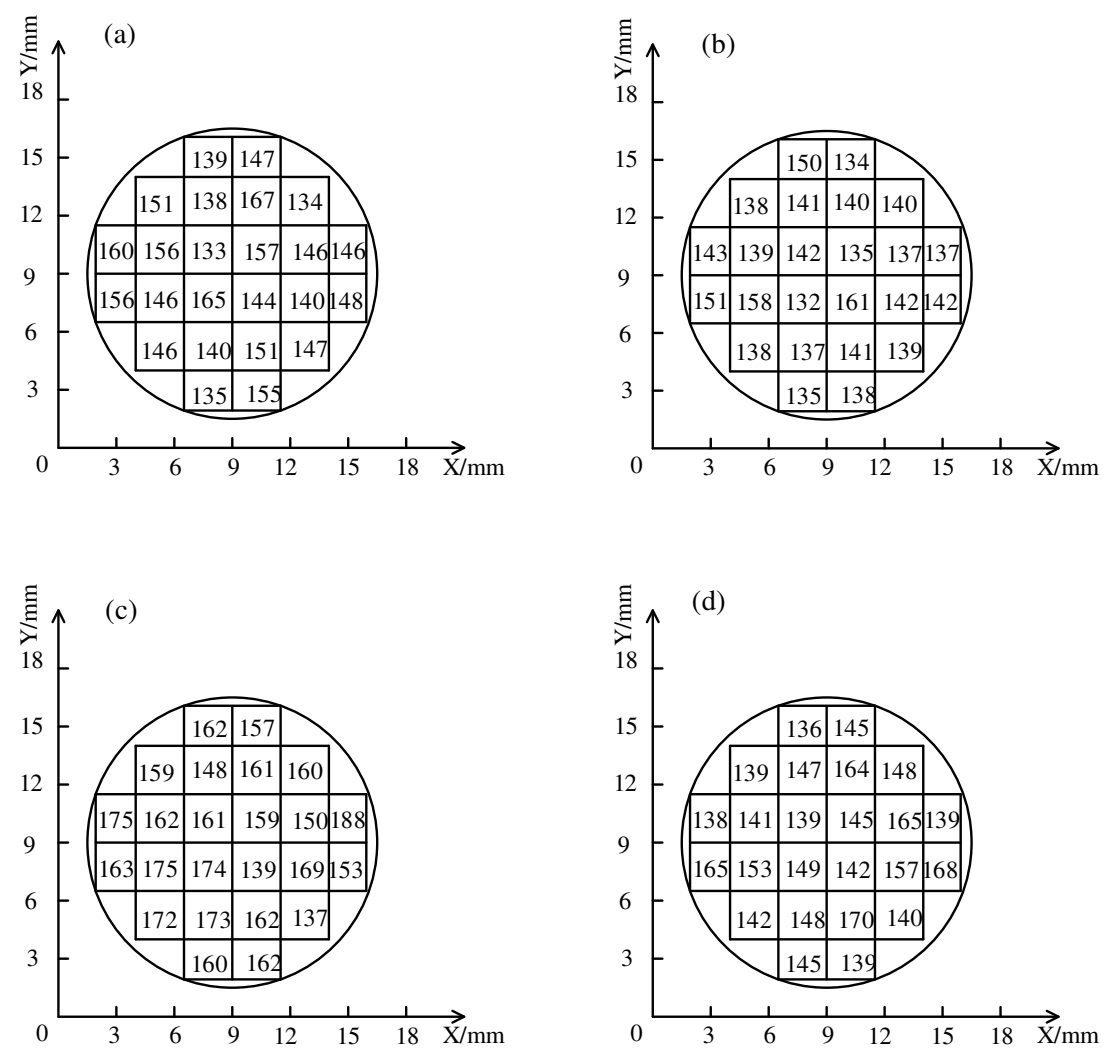

Fig.10 Distribution of surface roughness on the workpiece surface at a different rotational speed. (a) the upper surface, $n_{\mathrm{w}}=5 \mathrm{rpm}$. (b) lower surface, $n_{\mathrm{w}}=5 \mathrm{rpm}$. (c)the upper surface, $n_{\mathrm{w}}=6 \mathrm{rpm}$. (d) lower surface, $n_{\mathrm{w}}=6 \mathrm{rpm}$

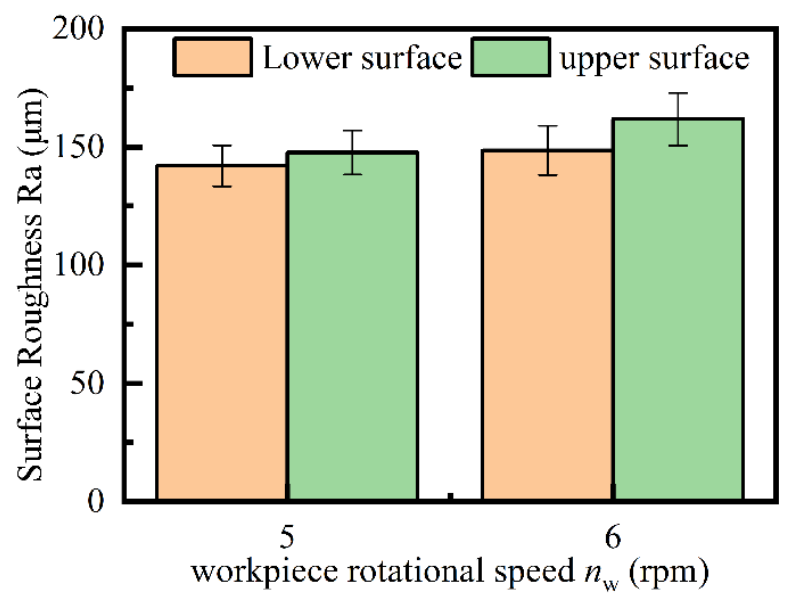

Fig.11 The effect of selected workpiece rotational speed on the surface roughness 


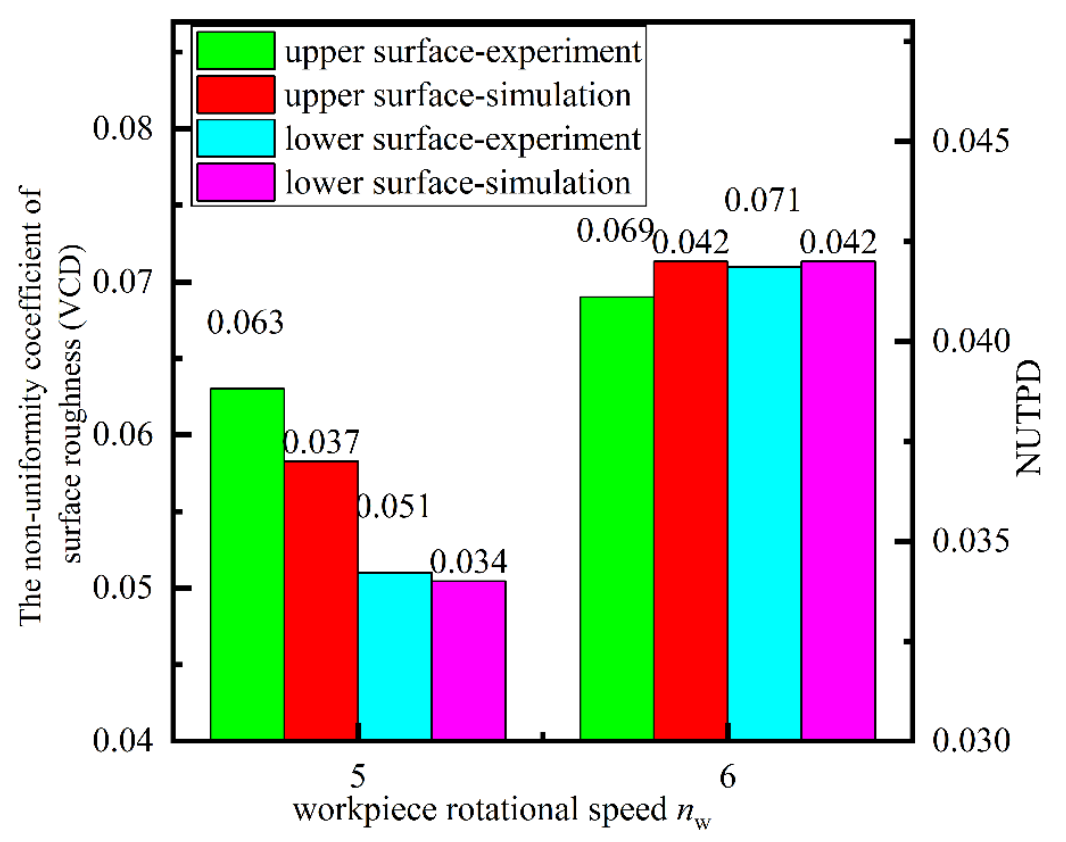

Fig.12 The effects of workpieces rotational speed on the distribution of trajectory point and roughness

\subsection{Surface Flatness}

$\mathrm{PV}$, as the most important parameter of the surface accuracy in the lapping process, is influenced significantly by the grit trajectory left on the workpiece. Fig.13 shows the value of PV after the process at selected workpiece rotational speed. The value of PV in the upper surface and the lower surface is $2.62 \mu \mathrm{m}$ and $2 \mu \mathrm{m}$ at $n_{\mathrm{w}}=5 \mathrm{rpm}$ respectively. However, they are $3.05 \mu \mathrm{m}$ and $2.74 \mu \mathrm{m}$ at $n_{\mathrm{w}}=6 \mathrm{rpm}$, respectively. Though the surface profile of the workpiece is similar at different rotational speeds, an obvious different result still exists. The smaller value of PV was achieved in both upper surface and lower surface at $n_{\mathrm{w}}=5 \mathrm{rpm}$. It can be seen from the simulation result above that the distribution of the grit on the surface of the workpiece is more even when the rotation speed of the workpiece is $5 \mathrm{rpm}$, so more uniform surface material removal can be achieved. In addition, the more obvious edge collapse on the workpiece surface occurs when the workpiece rotational speed is 6rpm. This is because the density of the grit trajectory point at the edge of the workpiece is greater than the density of the center of the workpiece. When more abrasive particles traverse the edge of the workpiece, the material removal from the edge of the workpiece is greater, which causes the deterioration of the flatness. However, when the workpiece rotational speed is $5 \mathrm{rpm}$, the track density distribution is generally uniform on the whole workpiece surface, which is advantaged to alleviate the deterioration of flatness. Therefore, when the workpiece speed is odd, a better flatness after machining can be obtained than when it is even. 


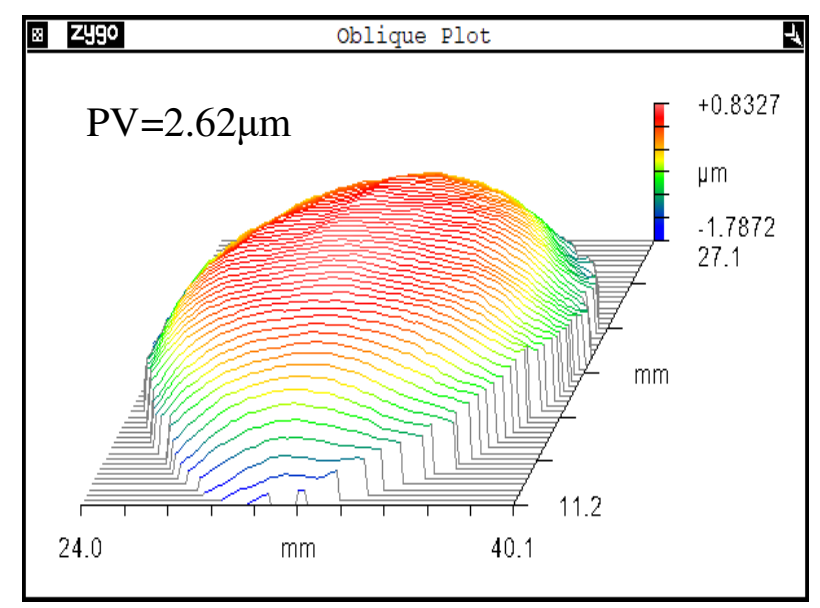

(a)

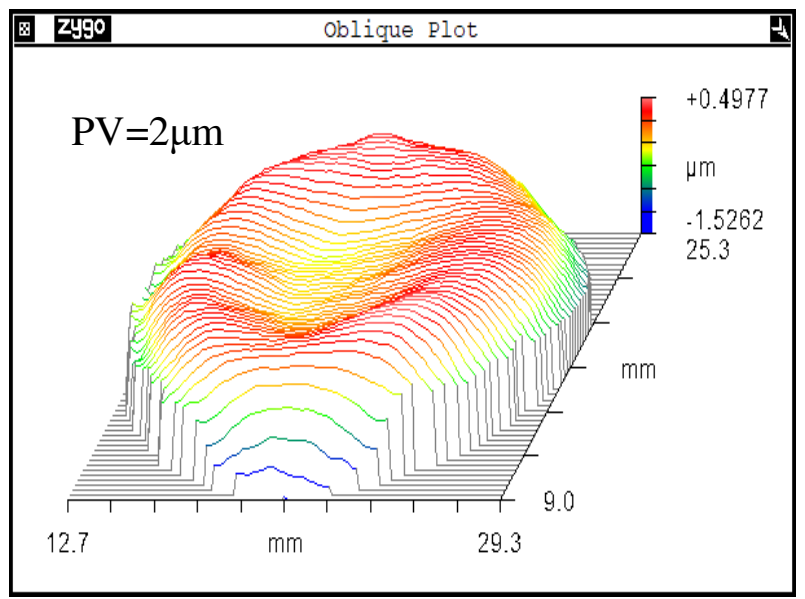

(b)

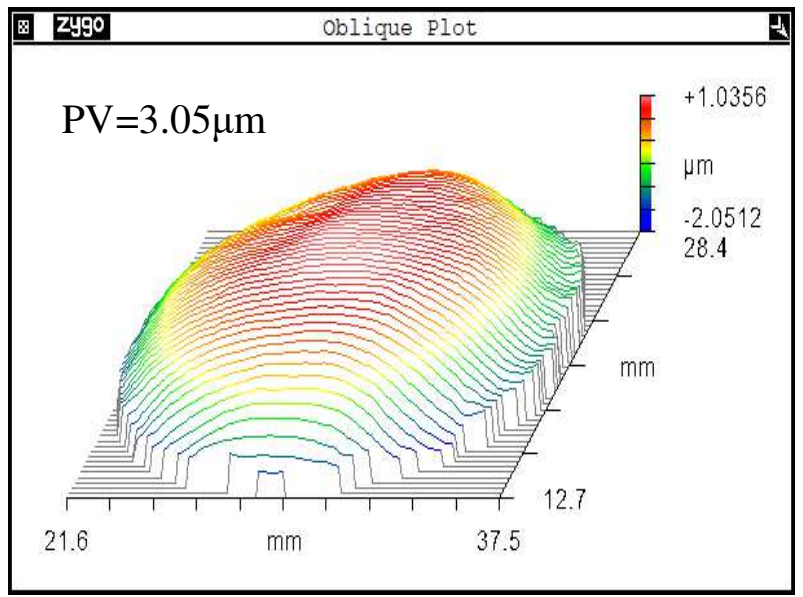

(c) 


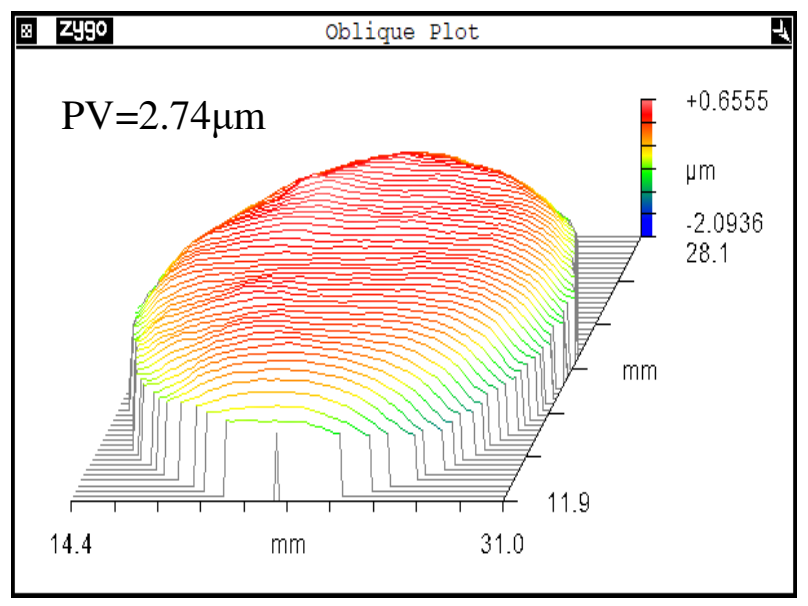

(d)

Fig.13 3D contour of the wafer surface with different rotation speeds. (a) the upper surface, $P V=2.62, n_{\mathrm{w}}=5 \mathrm{rpm}$. (b) lower surface, $\mathrm{PV}=2, n_{\mathrm{w}}=5 \mathrm{rpm}$. (c)the upper surface, $\mathrm{PV}=3.05, n_{\mathrm{w}}=6 \mathrm{rpm}$. (d) lower surface, $\mathrm{PV}=2.74, n_{\mathrm{w}}=6 \mathrm{rpm}$

\section{Conclusions}

To improve the uniformity of surface quality of the workpieces during the double-side planetary lapping, a mathematical model for calculating the grit trajectory left on the workpiece considering the workpiece rotation is established. Moreover, the influence of workpiece rotation speed on trajectory distribution is simulated through MATLAB software, and validated by conducting double-side planetary lapping experiments with YAG wafer at two typically selected rotational speeds. The main conclusions are:

1) By utilizing the method for simulating the trajectory of multiple grits, a mathematical model for calculating the grit trajectory left on the workpiece during the double-side planetary lapping was established. The NUTPD was introduced to evaluate the distribution uniformity of the grit trajectory on the workpiece, and the lower value of the NUTPD represents the more uniform the material removal, thus the better surface quality. The uniformity of the surface roughness was reflected by the value of VCR in the experiment, and the simulation result was validated by comparing the NUTPD and the VCR at different selected workpiece rotational speeds.

2) The influence of workpiece rotation speed on the values of the NUTPD presents a fluctuating characteristic. When $n_{\mathrm{w}}=1,3,5,7,9$, better flatness, and roughness after lapping can be obtained, and the edge-depression is smaller, due to the more even distribution of the grit trajectory in the center and edge of the wafer.

3) The optimized processing parameters can be used to guide the improvement of surface quality in the double-sided planetary lapping process. 


\section{Declarations}

\section{a. Funding}

National Natural Science Foundation of China (51775084)

\section{b. Competing interests}

Authors declare no competing interests

\section{c. Availability of data and material}

Data used to support the findings of this study are available from the corresponding author upon request.

\section{d. Code availability}

Not applicable

\section{e. Ethics approval}

Not applicable

\section{f. Consent to participate}

Not applicable

\section{g. Consent for publication}

Not applicable

\section{h. Authors' contributions}

Renke Kang contributed to the conception of the study;

Yufan Jia performed the experiment;

Xianglong Zhu contributed significantly to analysis and manuscript preparation;

Lei Yang performed the data analyses and wrote the manuscript;

Xiaoguang Guo helped perform the analysis with constructive discussions 


\section{References}

1. Campbell JH, Hayden JS, Marker A (2011) High-Power Solid-State Lasers: A Laser Glass Perspective. Int J Appl Glas Sci 2:3-29. https://doi.org/10.1111/j.2041-1294.2011.00044.X

2. Kang J, Chunzheng D, Jinxing K, Yi, C; Yunwen S, Shanglin W (2020) Prediction of clamping deformation in vacuum fixture-workpiece system for low-rigidity thin-walled precision parts using finite element method. Int J Adv Manuf Technol 109:1895-1916. https://doi.org/10.1007/s00170-020-05745-5

3. Han X, Jin Z, Mu Q, Mu Q, Niu N, Zhou Ping (2019) Internal stress and deformation analysis of ultra-thin plate-shaped optical parts in thinning process. Opt Express 27:27202. https://doi.org/10.1364/oe.27.027202

4. Komanduri R, Lucca DA, Tani Y (1997) Technological advances in fine abrasive processes. CIRP Ann Manuf Technol 46:545-596. https://doi.org/10.1016/S0007-8506(07)60880-4

5. Evans CJ, Paul E, Dornfield D, Lucca D.A, Byrne G, Tricard M, Klocke F, Dambon O, Mullany B.A (2003) Material removal mechanisms in lapping and polishing. CIRP Ann - Manuf Technol 52:611-633. https://doi.org/10.1016/S0007-8506(07)60207-8

6. Pan B, Kang R, Guo J, Fu H, Du D, Kong J (2019) Fabrication of thin copper substrate by double-sided lapping and chemical mechanical polishing. J Manuf Process 44:47-54. https://doi.org/10.1016/j.jmapro.2019.05.035

7. Yiqing Y, Zhongwei H, Wenshan W, Huan Z, Jing L, Xipeng X (2020) The double-side lapping of SiC wafers with semifixed abrasives and resin-combined plates. Int J Adv Manuf Technol 108:997-1006. https://doi.org/10.1007/s00170-019-04592-3

8. Uhlmann E, Ardelt T, Spur G (1999) Influence of kinematics on the face grinding process on lapping machines. CIRP Ann - Manuf Technol 48:281-284. https://doi.org/10.1016/S0007-8506(07)63184-9

9. Tseng W, Chin J, Kang L (1999) A Comparative Study on the Roles of Velocity in the Material Removal Rate during Chemical Mechanical Polishing. J Electrochem Soc 146:1952-1959.

https://doi.org/10.1149/1.1391872

10. Kim H, Jeong H (2004) Effect of Process Conditions on Uniformity of Velocity and Wear Distance of Pad and Wafer during Chemical Mechanical Planarization. J Electron Mater 33:53-60.

https://doi.org/10.1007/s11664-004-0294-4

11. Hocheng H, Tsai HY, Tsai MS (2000) Effects of kinematic variables on nonuniformity in chemical mechanical planarization. Int J Mach Tools Manuf 40:1651-1669.

https://doi.org/10.1016/S0890-6955(00)00013-4

12. Feng T (2007) Nonuniformity of wafer and pad in CMP: Kinematic aspects of view. IEEE Trans Semicond Manuf 20:451-463. https://doi.org/10.1109/TSM.2007.907625

13. Fang C, Zhao Z, Hu Z (2017) Pattern optimization for phyllotactic fixed-abrasive pads based on the trajectory method. IEEE Trans Semicond Manuf 30:78-85. https://doi.org/10.1109/TSM.2016.2637058

14. Fang C, Liu C, Zhao Z, Lin Y, Hu Z, Xu x (2018) Study on geometrical patterns of textured fixed-abrasive pads in sapphire lapping based on trajectory analysis. Precis Eng 53:169-178.

https://doi.org/10.1016/j.precisioneng.2018.03.008

15. Fang C, Zhao Z, Lu L, Lin Y (2017) Influence of fixed abrasive configuration on the polishing process of silicon wafers. Int J Adv Manuf Technol 88:575-584. https://doi.org/10.1007/s00170-016-8808-9

16. Hu Z, Fang C, Deng W, Zhao Z, Lin Y, Xu X (2017) Speed ratio optimization for ceramic lapping with fixed diamond pellets. Int J Adv Manuf Technol 90:3159-3169. https://doi.org/10.1007/s00170-016-9644-7

17. Cheng Z, Gao H, Liu Z, Guo D (2020) Investigation of the trajectory uniformity in water dissolution 
ultraprecision continuous polishing of large-sized KDP crystal. Int J Extrem Manuf 2:

https://doi.org/10.1088/2631-7990/abaabe

18. Liu Z, Jin Z, Wu D, Guo J (2019) Investigation on Material Removal Uniformity in Electrochemical Mechanical Polishing by Polishing Pad with Holes. ECS J Solid State Sci Technol 8:P3047-P3052. https://doi.org/10.1149/2.0071905jss

19. Piotrowski N (2020) Tool wear prediction in single-sided lapping process. Machines 8:1-11. https://doi.org/10.3390/machines8040059

20. Liao D, Xie R (2017) A kinematic model for material removal distribution and surface figure in the full-aperture polishing. ISAAT 2017 - Proc 20th Int Symp Adv Abras Technol 57:761-770. https://doi.org/10.1364/ao.57.000588

21. Dong Z, Cheng H (2014) Study on removal mechanism and removal characters for SiC and fused silica by fixed abrasive diamond pellets. Int J Mach Tools Manuf 85:1-13. https://doi.org/10.1016/j.ijmachtools.2014.04.008

22. Zhao D, Wang T, He Y, Lu X (2013) Kinematic optimization for chemical mechanical polishing based on a statistical analysis of particle trajectories. IEEE Trans Semicond Manuf 26:556-563.

https://doi.org/10.1109/TSM.2013.2281218

23. Lee C, Wang C, Wa D, Shi H (2019) Optimal analysis of trajectory parameters for the double-sided polishing in planetary motion mode. 11. https://doi.org/10.1117/12.2541653

24. Wang L, Hu Z, Fang C, Yu Y, Xu X (2018) Study on the double-sided grinding of sapphire substrates with the trajectory method. Precis Eng 51:308-318. https://doi.org/10.1016/j.precisioneng.2017.09.001

25. Hashimoto Y, Sano T, Furumoto T, Hosokawa A (2019) Estimation of material removal rate distribution in double-sided polishing of thick square workpiece considering workpiece attitude. J Adv Mech Des Syst Manuf 13:1-12. https://doi.org/10.1299/jamdsm.2019jamdsm0020

26. Jing Y, Li W, Hu G, Hu X (2005) Motion analysis for double-sided polishing process and mathematical model establishing. J Nanj U of Aero and Astro, 86-89. https://10.3969/j.issn.1005-2615.2005.z1.019

27. Satake U, Enomoto T, Fujii K, Hirose K (2016) Optimization Method for Double-sided Polishing Process based on Kinematical Analysis. Procedia CIRP 41:870-874. https://doi.org/10.1016/j.procir.2015.12.043 